Title of Manuscript: Nursing informatics and coaching based interventions: A

protocol for delivery

\title{
Author:
}

Suzanne Fredericks RN, PhD

Associate Professor

Daphne Cockwell, School of Nursing, Ryerson University

350 Victoria St.

Toronto, ON; M5B 2K3

Office Number: 416-979-5000 ext. 7978; sfrederi@ ryerson.ca

Fax Number: 416-979-5332

Biographical Statement: Dr. Fredericks is an Associate Professor at Ryerson University. Her program of research focuses on supporting patients following invasive procedures. Dr. Fredericks has led several quantitative focused studies and has served as author and co-author on numerous manuscripts. 
Introduction

Coronary Artery Bypass Graft (CABG) and Valve Replacement (VR) are the most common surgical treatments for cardiovascular disease (Cardiac Care Network of Ontario (CCNO), 2007). Despite their advantages, CABG and VR result in changes in the physical and psychological functioning of individuals within the first three weeks following surgery (Cebeci \& Celik, 2008). This gives rise to an increased demand for the performance of specific behaviours post-discharge aimed at enhancing self-management within the home environment (Barnason, Zimmerman, Anderson, Mohr-Burt, Nieveen, 2000; CCNO; Watt-Watson \& Stevens, 1998). Thus, patients who have had CABG and/or VR need to have a good understanding of how to engage, as well as perform behaviours to prevent the onset of post-operative complications. One way in which this can be achieved is through coaching based interactions.

Coaching is a motivational approach often used to encourage the implementation of self-management educational instruction (Vale et al., 2002). It encompasses the processing, management, and retrieval of information (Vale et al., 2002). The outcome of coaching is to enhance patients' knowledge and improve the health of individuals by optimizing information management and communication (Vale et al.). Coaching has been shown to be effective across the cardiac population in promoting target cholesterol within the home environment (Vale et al.). However, coaching is not a common interaction routinely used in nursing care. This may be due to unfamiliarity with the interaction and/or lack of understanding of how to engage in coaching behaviours within the clinical setting. The purpose of this discursive paper is to present a description of a protocol for delivering a coaching based intervention to patients following heart surgery and its implications for 
nursing informatics. The paper will begin with a brief overview of coaching followed by a detailed, step-by-step outline of a coaching based intervention that can be applied to patients following $\mathrm{CABG}$ and/or VR surgery. The implications for nursing informatics associated with incorporating this intervention into standard of care are described.

Description of coaching as a self-management based educational interaction Coaching is usually provided by a trained therapist who has experience working with patients from a specific area of interest (i.e. cardiovascular surgery). The selfmanagement approach to coaching is delivered in 4 stages, ranges between 20-30 minutes in duration, and is delivered at 2 points in time. The first stage involves asking questions to establish patient's knowledge about specific behaviours in which they are required to perform during their home recovery period. Having an understanding of previous selfmanagement knowledge provides a context for evaluating the successes and difficulties, as well as level of understanding and misunderstanding related to the content. In addition, information related to reasons as to why engagement in recommended behaviours was not performed is also collected. The second stage of the coaching interaction consists of behaviour reinforcement in which misconceptions are clarified and self-management information is presented in greater depth. Stage 3 consists of problem solving and motivational guidance in which the therapist works with the patient to identify personal barrier that prevent performance of self-management behaviours. The therapist works with the patient to problem solve these barriers, negotiate realistic goals, and brainstorm creative, concrete, and realistic strategies to being able to perform selfmanagement behaviours within the home environment. During the final stage of the coaching session, when the interaction is first delivered, the therapist works with the 
patient to establish timelines for completion of goals. On the second delivery of the interaction at 2 weeks following hospital discharge, during stage 4 of the coaching interaction, the therapist assesses whether or not established goals have been achieved. The therapist also provides positive encouragement, praise, and support for efforts and relapses (Vale et al., 2002).

The following is a detailed, step-by-step description of a protocol for delivering a coaching based intervention to patients following CABG and/or VR surgery.

The goal of self-management coaching sessions is to enhance knowledge by assisting patients in their performance of routine activities during their home recovery. To this end, a therapist trained in the delivery of coaching interactions is used to deliver the self-management support treatment. The coaching session is usually completed via a combination of media that include: in person, over the telephone, or via online chat discussion forum. If an online chat is to be used in the coaching interaction, then a secure chat room is created for each patient to ensure privacy.

In spite of the medium in which the coaching is provided, the general format for the sessions remains the same. To begin, the therapist asks the patient questions about their knowledge of specific self-management behaviours that they are required to perform at home to manage post-operative $\mathrm{CABG}$ and/or VR complications. The behaviours are based on a review of the literature and address the following topics that include: symptom management (i.e. incision and chest pain, nausea, vomiting, fatigue, sleep disturbance, constipation), edema/water retention, nutrition, medication management, pulmonary and abdominal complications, and performance of usual activity. A multiple choice response format is typically used to assess the total score, which would be represented by the total 
number of correct responses. During the interaction, the correct response for each behaviour is identified.

During the second stage of the self-management coaching interaction the therapist begins the education reinforcement section by reviewing self-management behaviours patients who have received CABG and/or VR are expected to perform following hospital discharge. The identical content that was addressed in stage 1 is repeated in this stage. However, instead of testing the individual's knowledge, the patient is provided detailed information related to the specific area in which they did not score a correct response.

Each content area is broken down into subsections to further explain the item. In particular, complications focus on how to recognize complications and addresses the signs and symptoms associated with 3 most common post-operative CABG/and VR complications that include pulmonary, wound, and abdominal complications. Strategies to decrease or avoid complications during the post-operative recovery period are also presented and include use of an incentive spirometer and deep breathing and coughing exercises. As well, techniques to decrease or avoid wound complications that include taking daily showers with soap and water; not using ointments or lotions near or on the incision; use of a dry, sterile dressing to draining areas; and the need to contact physician if incision is draining a white, yellow, or dark red, thick substance. Furthermore, notifying patients of the need to contact the family physician or going to the nearest emergency department immediately if stomach complications arise is also addressed throughout coaching session. With regards to activity, patients are instructed as to the appropriate physical activities that they should be performing during the first 3 weeks following hospital discharge. In particular, content related to walking, performance of 
usual activity (i.e. bathing, dressing, cooking, etc...), climbing stairs, lifting or pulling objects, and sexual activity is reviewed with the patient. The content that addresses medication management relate to strategies to enhance management of medication and include making sure that prescriptions are refilled in a timely manner and techniques are in place for patients to remember to take their pills. As well, an emphasis on the need to contact the pharmacist or family physician if side-effects are experienced and the importance of not missing taking medication or doubling up on missed doses is also reviewed. Finally, management of edema focuses on medication management, restriction of fluids, need to weigh self on a daily basis, reducing salt intake, elevating legs while sitting, and contacting physician if symptoms associated with use of stockings occur such as: dryness, itchiness, redness, or numbness in feet.

The education material to be used is based on an extensive empirical review to ensure relevance to $\mathrm{CABG}$ and/or VR patients. Furthermore, the educational material is reviewed by the team of study collaborators. As the therapist proceeds through the education reinforcement section, any misconceptions are clarified and specific selfmanagement information is presented in greater depth.

The third stage of the coaching intervention involves problem solving and motivational guidance. Within this stage, patients are asked to identify personal barriers and facilitators to lifestyle change; problem-solve; negotiate realistic goals; and brainstorm creative, concrete, and realistic strategies for change. The following questions are asked: a) Are there any barriers that might prevent you from following the teaching related to activity performance (i.e. climbing stairs, walking, lifting objects, and avoiding strain)? b) Are there any barriers that might prevent you from following the teaching 
related to nutrition (i.e. drinking no more than 2 cups of fluid in a day, weighing oneself on a daily basis, and contacting physician if weight gain is more than $2 \mathrm{lbs}$ )? c) Are there any barriers that might prevent you from following the teaching related to medication management (i.e. taking your medication at the right time and on the correct day, refilling medication (s) when needed, and using a system to help with medication management)? d) Are there any barriers that might prevent you from following the teaching related to managing and preventing complications (i.e. performing deep breathing and coughing exercises as recommended, using the incentive spirometer as required as well, cleansing of incisional wounds, assessment of incisional wounds for signs of complications, and contacting physician when signs of complications are noted)?

During the final stage of the coaching session, timelines are established and goals are assessed. The therapist provides positive encouragement, praise, and support for efforts and relapses. At the end of this stage, the patient is reminded that there identified goals and timelines will be reviewed and reassessed by the therapist, who will contact them in two weeks.

\section{Implications for nursing informatics}

Even though coaching sessions can be delivered on line via chat room discussion, this medium is rarely used. This may be due to the demographic characteristics of patients who undergo CABG and/or VR. Over the past 10 years, a shift towards performing heart surgery on patients older than 65 years of age has occurred (Fasken, Wipke-Tevis, Sagehorn, 2001). Specifically, the average age of patients who undergo cardiovascular surgery is 68.9 years (Fredericks, Lo, Ibrahim, Leung, 2010). Similarly, advances in technology have provided alternatives to the delivery of coaching or support 
based interventions. However, the medium for delivering these interventions in the cardiovascular surgical (CVS) setting has predominantly remained the same, in that telephone interactions are most commonly used. Current evidence suggests that using a variety of mediums during patient interaction sessions enhances knowledge acquisition and behavioural change (Fredericks, Guruge, Sidani, Wan, 2010). However, the relationship between older individuals and the use of technology has not been clearly identified. Specifically, findings by Koch \& Hägglund (2009) suggest that research on health informatics in older individuals is still in its infancy and requires significant investigation.

In order to enhance the application of technology in the delivery of coaching or support based interactions in the CVS environment, continued investigation to assess patients' needs for technology and desirability to use technology as a tool to assist in their post-operative recovery experience is needed. Based on the results of a needs assessment, if patients indicate that they have minimal proficiency or do not value technology; then alternative mediums for delivering support based interventions should be offered. However, if patients are open to engaging in interactions through the use of technology then this mediums should be incorporated into the design of patient care.

Using existing technology (i.e. online chat rooms) to deliver coaching or support based interventions will allow patients the flexibility to interact with a nurse, in real time, from the comfort of their homes. They can review the transcripts from the coaching sessions anytime as it is stored on the online chat discussion boards, and they do not have to be proficient in using the internet to be successful. 
Future nursing research should assess the effectiveness of providing patients with the option to receive a coaching session through either telephone interaction or online chat rooms. Patient's preference for the medium of delivery of a coaching session will determine how they receive the intervention. Following delivery of the intervention, an assessment of acquisition of desired goals should be conducted to determine the effectiveness of the methodology (allowing patients the option to choose - i.e. patient preference). It is anticipated that providing patients with the option to choose to either use or not use technology during coaching sessions will enhance self-management of behaviours following heart surgery. 


\section{References}

Barnason, S., Zimmerman, L., Anderson, A., Mohr-Burt, S., \& Nieveen, J. (2000). Functional status outcomes of patients with a coronary artery bypass graft over time. Heart and Lung: The Journal of Acute and Critical Care, 29(1), 33-46.

Cardiac Care Network of Ontario (2007) Consensus panel on target setting. http://www.ccn.on.ca/index.cfm?fuseaction=sp\&sp=29, access June 2011. (accessed 23 June 2010).

Cebeci, F., \& Çelik, S. (2008). Discharge training and counseling increase self-care ability and decrease post-discharge problems in CABG patients. Journal of Clinical Nursing, 17, 412-420.

Fasken, L. L., Wipke-Tevis, D. D., \& Sagehorn, K. K. (2001). Factors associated with unplanned readmission following cardiac surgery. Progress in Cardiovascular Nursing, 3, 107-115.

Fredericks, S., Guruge, S., \& Sidani, S., Wan, T. (2010). Post-operative patient education: A systematic review. Clinical Nursing Research: An International Journal, 19, 2, 144-164.

Fredericks, S., Lo, J., Ibrahim, S., \& Leung, J. (2010). An examination of the difference in performance of self-care behaviours between white and non-white patients following CABG surgery: A secondary analysis. Canadian Journal of Cardiovascular Nursing, 20(4), 21-29.

Koch, S. \& Hägglund, M. (2009). Health informatics and the delivery of care to older people. Maturitas, 63(3), 195-199. 
Vale, M. J., Jelinek, M. V., Best, J. D., \& Santamaria, J. D. (2002). Coaching patients with coronary heart disease to achieve the target cholesterol: A method to bridge the gap between evidence-based medicine and the "real world" - randomized controlled trial. Journal of Clinical Epidemiology, 55, 245-252.

This manuscript has been published by the Canadian Journal of Nursing Informatics; Fredericks, S. (April 2012). Nursing informatics and coaching based interventions: A protocol for delivery. Canadian Journal of Nursing Informatics. (Featured Article), 6, 4. Available online at: https://tinyurl.com/7k47ktj. http://tinyurl.com/7k47ktj 